\title{
Letter to the Editor \\ Decreased blood flow in the right insula and middle temporal gyrus predicts negative formal thought disorder in schizophrenia
}

Stephanie Winkelbeiner*/1/a/d, Marialuisa Cavelti ${ }^{1 / a / b}$, Andrea Federspiel ${ }^{\mathrm{a}}$, Katharina Kunzelmann ${ }^{\mathrm{a}}$, Thomas Dierks ${ }^{\mathrm{a}}$, Werner Strik ${ }^{\mathrm{a}}$, Helge Horn ${ }^{\mathrm{a} / \mathrm{c}}$, Philipp Homanª/d

\footnotetext{
${ }^{1}$ Shared first authorship: The authors contributed equally to this work.

a Translational Research Center, University Hospital of Psychiatry and Psychotherapy, University of Bern, 3000 Bern 60, Switzerland.

${ }^{\mathrm{b}}$ Orygen, The National Centre of Excellence in Youth Mental Health \& Centre for Youth Mental Health, University of Melbourne, Parkville, VIC 3052, Australia.

${ }^{\mathrm{c}}$ Institute for Psychiatry and Psychotherapy Bern, Waisenhausplatz 25, 3011 Bern, Switzerland.

${ }^{\mathrm{d}}$ Center for Psychiatric Neuroscience, The Feinstein Institute for Medical Research, Zucker School of Medicine at Northwell/Hofstra, New York, NY, USA.

* Corresponding author: stephanie.winkelbeiner@upd.unibe.ch; phone: +1 3473251630
}

Word counts of the text body: $776(800)$ 


\section{Dear Editors,}

Formal thought disorder (FTD) causes severe disturbances of language and communication, and affects $50 \%-80 \%$ of people with schizophrenia (Roche et al., 2015). It is part of the disorganization syndrome that has been found to distinguish patients with schizophrenia from patients with psychotic bipolar disorder, and may, thus, inform differential diagnosis (Palaniyappan et al., 2018). The breakdown of effective communication in FTD is associated with structural and functional alterations of the fronto-temporal language network (Cavelti et al., 2018; Horn et al., 2009; Kircher et al., 2008; Palaniyappan et al., 2015; Strik et al., 2017; Sumner et al., 2017) but might extend to areas outside this network including the amygdala (Cavelti et al., 2018; Spalletta et al., 2010; Sumner et al., 2017), hippocampus (Cavelti et al., 2018; Kircher et al., 2008; Spalletta et al., 2010; Sumner et al., 2017), and insula (Cavelti et al., 2018; Horn et al., 2009; Palaniyappan et al., 2015; Sumner et al., 2017). This range of FTDassociated brain areas might be partly due to the variety of symptoms subsumed under "FTD". Thus, a differentiation into potential subtypes such as positive FTD (i.e., unanticipated, bizarre, or inappropriately expressed speech) and negative FTD (i.e., speech reduced in quantity, content, or fluency) as suggested by factor analytical studies might be more accurate (Roche et al., 2015). Positive FTD has been associated with decreased cortical thickness of the superior temporal gyrus (STG) (Horn et al., 2009), increased activity in the middle temporal gyrus (MTG) (Kuperberg et al., 2007), and decreased volume of the amygdala (Spalletta et al., 2010) and hippocampus (Kircher et al., 2008; Spalletta et al., 2010), whereas negative FTD has been associated with decreased grey matter volume in areas including the MTG and insula (Palaniyappan et al., 2015). However, no study to date has investigated both structural and functional measures to determine if positive and negative FTD reflect two neurophysiologically distinct entities. Therefore, we examined cortical thickness, subcortical volume, and blood 
perfusion of language (MTG and STG) and language-associated brain areas (amygdala, hippocampus, and insula) as predictors of positive and negative FTD in schizophrenia.

We included twenty-four right-handed and German-speaking patients with a schizophrenia spectrum disorder according to the DSM-IV-TR between 18 and 65 years of age. Severity of FTD was assessed using the Thought, Language, and Communication (TLC) scale, with the subscales Disorganization and Emptiness providing measures of positive and negative FTD. All subjects gave written informed consent. The study was approved by the ethics committee of the Kanton Bern, Switzerland (see the Supplementary Material for details on the study sample and comparisons with healthy controls).

A 3T Magnetom Verio system (Siemens Erlangen, Germany) was used to create the highresolution 3D T1-weighted images with a gradient echo and the absolute cerebral blood flow $(\mathrm{CBF})$ images with pseudo continuous arterial spin labeling. Structural images were processed using Freesurfer 5.1.0, perfusion images were analyzed using FSL 5.0. Mean regional CBF (rCBF), cortical thickness, and subcortical volume values were extracted for every region of interest (see Supplementary Figure 1). We used multivariable linear regression analyses in R 3.1.3 with the TLC Total score $(n=24)$, Emptiness score $(n=16)$, and Disorganization score $(n=16)$ as dependent variables, the normalized rCBF, cortical thickness, and subcortical volume as independent variables, and age and whole brain global cortical thickness or subcortical volume, respectively, as covariates. The missing values for the TLC subscales were due to missing single items, while the total scores were obtained for all participants. Results were corrected to adjust for false discovery rate (FDR) and considered significant for $p<0.05$ (see the Supplementary Methods for more details).

Negative FTD (TLC Emptiness) was significantly correlated with decreased rCBF in the right insula (FDR-corrected $p=0.031$; Figure 1) and in the right MTG (FDR-corrected $p=0.022$; 
Figure 1). Positive FTD (TLC Disorganization) did not significantly correlate with structural or metabolic measures $(p>0.05$; Figure 1$)$.

This study provided evidence that metabolic changes in the right insula and MTG contribute to negative FTD in schizophrenia. This finding might be best understood in the light of the disconnection hypothesis which suggests that a perturbed lateralization of usually left-sided language areas leads to more diffuse language processing (Kircher et al., 2008). As the insula is also involved in the integration and sequencing of information into a stringent stream of consciousness (Strik et al., 2017), the decreased rCBF found in this region could explain deficient planning of utterances of speech underlying FTD. Yet, we did not find evidence for structural correlates of negative FTD, as well as structural or metabolic correlates of positive FTD. While this is in contrast to previous studies (Horn et al., 2009; Kircher et al., 2008; Kuperberg et al., 2007; Palaniyappan et al., 2015; Spalletta et al., 2010), it is in line, nonetheless, with frequently reported null findings (for a review see Sumner et al. (2017)). The most likely explanation is the small sample size of the current study that limits a conclusive decision whether the non-significant findings actually reflect the absence of effects altogether or whether the effects were relevant but too small to be detected.

While this study provides preliminary evidence that abnormal blood perfusion contributes to negative FTD, studies investigating larger samples are needed to elucidate whether positive and negative FTD effectively reflect distinct entities of FTD with different neurophysiological characteristics. 
A

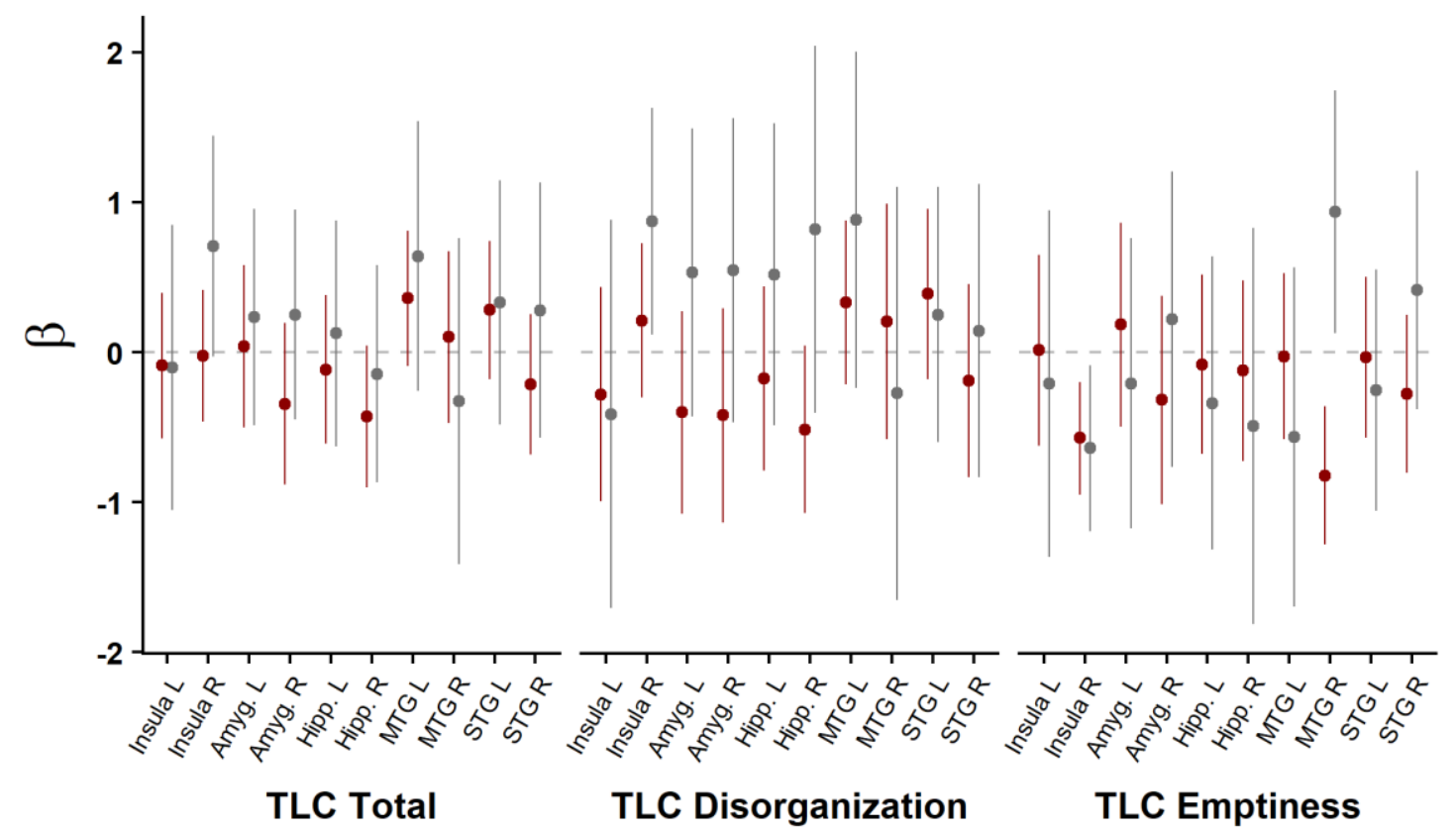

$\bullet$ Perfusion $\bullet-$ Cortical thickness, subcortical volume

\begin{tabular}{clllll}
\hline & $\boldsymbol{\beta}$ & \multicolumn{2}{c}{ CI } & $\boldsymbol{p}$ & $\boldsymbol{p}$ \\
& & $\mathbf{2 . 5 \%}$ & $\mathbf{9 7 . 5 \%}$ & (uncorrected) & (FDR-corrected) \\
\hline $\begin{array}{c}\text { TLC Emptiness (per 10 points increase) } \\
\text { rCBF in insula, right }\end{array}$ & & & & & \\
rCBF in MTG, right & -0.57 & -0.95 & -0.20 & $0.006^{* *}$ & $0.031^{*}$ \\
& -0.82 & -1.28 & -0.36 & $0.002^{* *}$ & $0.022^{*}$ \\
\hline
\end{tabular}

Abbreviations. CI, confidence interval; rCBF, regional cerebral blood flow; FDR, false discovery rate; MTG, middle temporal gyrus; TLC, Thought, Language, and Communication scale.

Multivariate linear regression; Significance at: *, $p<0.05 ;{ }^{* *}, p<0.01$.

Figure 1. A priori defined regions of interest (ROI) with cortical thickness or subcortical volume and regional cerebral blood flow (rCBF). A. Standardized regression coefficients with $95 \%$ confidence intervals are shown. Mean beta values (dots) represent the partial correlations (positive $>0$; negative $<0$ ) of regional cerebral blood flow (perfusion; red), cortical thickness, and subcortical volume (gray) in the ROIs (amygdala (Amyg.), hippocampus (Hipp.), insula, middle temporal gyrus (MTG), and superior temporal gyrus 
(STG)) with formal thought disorder (FTD). Confidence intervals not touching the zero line indicate an uncorrected significant effect at $p<0.05$. B. Significant results of multivariable linear regression analyses. Partial correlations of the Thought, Language, and Communication (TLC) scores for negative FTD (TLC Emptiness) with metabolic measures before and after false discovery rate (FDR)-correction. 


\section{Funding}

This research has been supported by grants from the Swiss National Science Foundation (\#320030_146789,\#152619,\#127359). 


\section{References}

Cavelti, M., Kircher, T., Nagels, A., Strik, W., Homan, P., 2018. Is formal thought disorder in schizophrenia related to structural and functional aberrations in the language network? A systematic review of neuroimaging findings. Schizophrenia research.

Horn, H., Federspiel, A., Wirth, M., Mueller, T.J., Wiest, R., Wang, J.J., Strik, W., 2009. Structural and Metabolic Changes in Language Areas Linked to Formal Thought Disorder. Br J Psychiatry 194, 130-138.

Kircher, T., Whitney, C., Krings, T., Huber, W., Weis, S., 2008. Hippocampal dysfunction during free word association in male patients with schizophrenia. Schizophrenia Research 101(1), 242-255.

Kuperberg, G.R., Deckersbach, T., Holt, D.J., Goff, D., West, W., 2007. Increased temporal and prefrontal activity in response to semantic associations in schizophrenia. Archives of General Psychiatry 64(2), 138-151.

Palaniyappan, L., Deshpande, G., Lanka, P., Rangaprakash, D., Iwabuchi, S., Francis, S., Liddle, P.F., 2018. Effective connectivity within a triple network brain system discriminates schizophrenia spectrum disorders from psychotic bipolar disorder at the single-subject level. Schizophrenia research.

Palaniyappan, L., Mahmood, J., Balain, V., Mougin, O., Gowland, P.A., Liddle, P.F., 2015. Structural correlates of formal thought disorder in schizophrenia: An ultra-high field multivariate morphometry study. Schizophr Res 168(1-2), 305-312.

Roche, E., Creed, L., MacMahon, D., Brennan, D., Clarke, M., 2015. The Epidemiology and Associated Phenomenology of Formal Thought Disorder: A Systematic Review. Schizophr Bull 41(4), 951-962. 
Spalletta, G., Spoletini, I., Cherubini, A., Rubino, I.A., Siracusano, A., Piras, F., Caltagirone, C., Marini, A., 2010. Cortico-subcortical underpinnings of narrative processing impairment in schizophrenia. Psychiatry Research: Neuroimaging 182(1), 77-80.

Strik, W., Stegmayer, K., Walther, S., Dierks, T., 2017. Systems Neuroscience of Psychosis: mapping schizophrenia symptoms onto brain systems. Neuropsychobiology 75(3), 100-116. Sumner, P.J., Bell, I.H., Rossell, S.L., 2017. A systematic review of the structural neuroimaging correlates of thought disorder. Neuroscience \& Biobehavioral Reviews. 